\title{
Spontaneous dislodgement of a biliary tumor in a patient with hepatocellular carcinoma
}

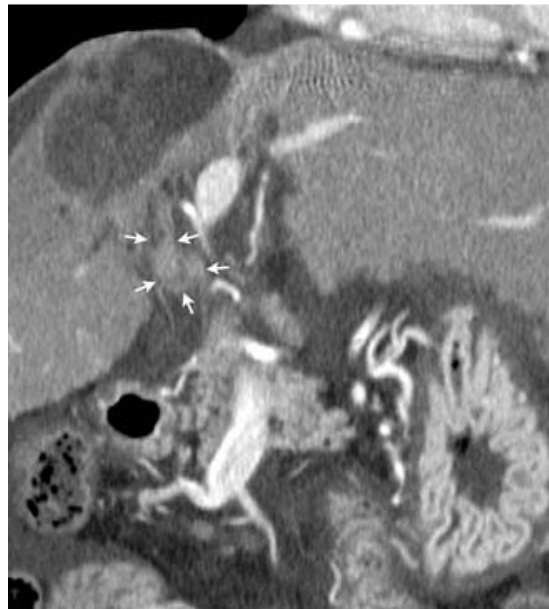

Fig. 1 Computed tomography (CT) showed a 15-mm hypervascular tumor in the common hepatic duct adjacent to the area previously treated with radiofrequency ablation (RFA) (arrows).

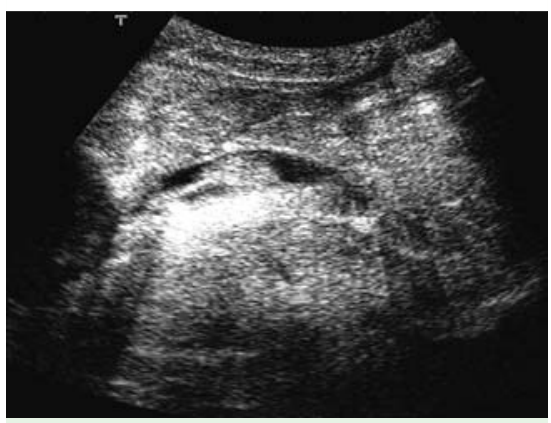

Fig. 2 Contrast-enhanced ultrasonography with Sonazoid showed a hypervascular pedunculated tumor in the upper extrahepatic bile duct.

A 72-year-old woman with Child-Pugh B cirrhosis was hospitalized in our department for transarterial chemoembolization (TACE) for the recurrence of hepatocellular carcinoma with biliary invasion. She had undergone radiofrequency ablation (RFA) therapy 1 year earlier. Contrast-enhanced computed tomography (CT) showed a 15-mm hypervascular tumor in the common hepatic duct adjacent to the area previously treated with RFA (๑ Fig. 1).

Subsequent contrast-enhanced ultrasonography with Sonazoid showed a hypervascular pedunculated tumor in the upper extrahepatic bile duct ( $\bullet$ Fig. 2 ).

On admission, the patient was asymptomatic with normal serum bilirubin and
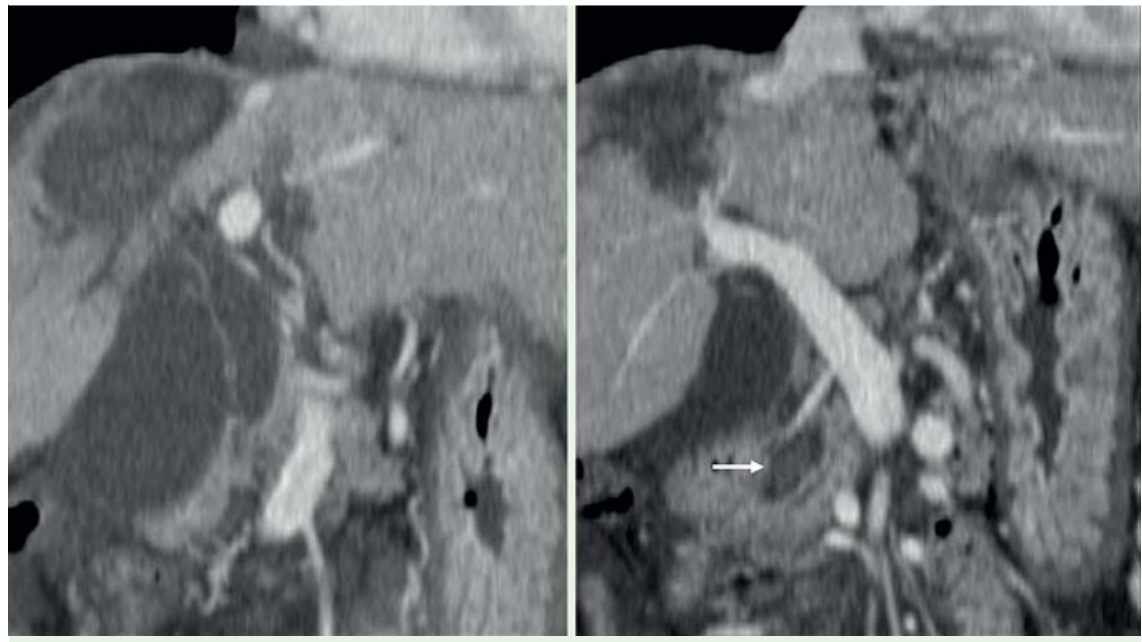

Fig. 3 Computed tomography (CT) revealed that: a the tumor in the common hepatic duct had disappeared, and $\mathbf{b}$ a lesion with slightly high density (arrow) had appeared in the lower part of the common bile duct instead.

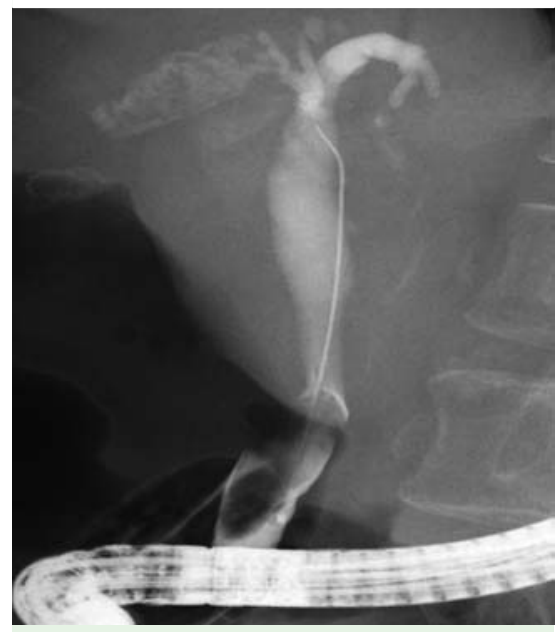

Fig. 4 A cholangiogram showed a $9 \times 30-\mathrm{mm}$ filling defect in the distal common bile duct.

biliary enzyme levels. In the morning when TACE was scheduled, however, she developed epigastralgia and vomiting, with elevated serum bilirubin and biliary enzyme levels. CT was performed immediately; the tumor in the common hepatic duct had disappeared, and a lesion with somewhat high density had appeared in the lower part of the common bile duct instead ( Fig. 3).

We suspected that the biliary tumor thrombus had spontaneously migrated to the lower common bile duct and was causing her symptoms. Emergent endo-

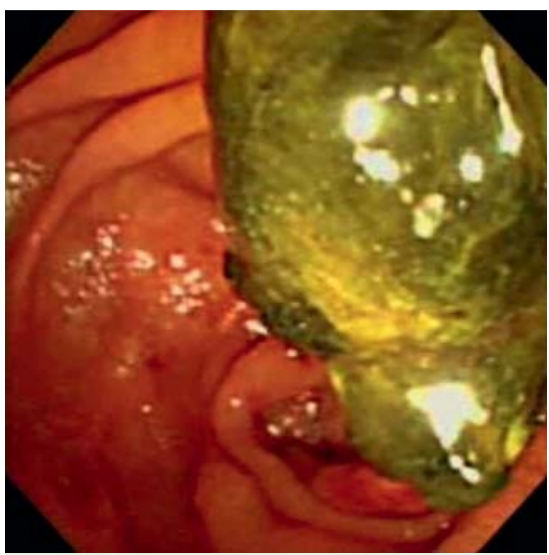

Fig. 5 An endoscopic image showed the necrotic mass of hepatocellular carcinoma.

scopic retrograde cholangiopancreatography showed a $9 \times 30-\mathrm{mm}$ filling defect in the distal common bile duct ( $\bullet$ Fig. 4).

After endoscopic papillary balloon dilation with a $10-\mathrm{mm}$ balloon, a blackish green tissue was obtained using a retrieval basket catheter ( $\bullet$ Fig. 5, $\diamond$ Video 1 ). Histopathological examination revealed hepatocellular carcinoma with extensive necrosis.

\section{Video 1}

After endoscopic papillary balloon dilation, the biliary tumor thrombus was removed using a retrieval basket catheter. 
Endoscopy_UCTN_Code_CCL_1AZ_2AC

Competing interests: None

H. Kogure, K. Miyabayashi, T. Tsujino, H. Isayama, R. Tateishi, K. Koike Department of Gastroenterology, Graduate School of Medicine, University of Tokyo, Japan
Bibliography

DOI $10.1055 / \mathrm{s}-0030-1256320$

Endoscopy 2011; 43: E232 -E233

(c) Georg Thieme Verlag KG Stuttgart · New York . ISSN 0013-726X
Corresponding author H. Kogure, MD

Department of Gastroenterology Graduate School of Medicine

University of Tokyo

7-3-1 Hongo, Bunkyo-ku

Tokyo 113-8655

Japan

Fax: +81-3-38140021

kogureh-tky@umin.ac.jp 